\title{
Shades of emotion: What the addition of sunglasses or masks to faces reveals about the development of facial expression processing
}

\author{
Debi Roberson $^{\mathrm{a}, *}$, Mariko Kikutani $^{\mathrm{a}}$, Paula Döge ${ }^{\mathrm{b}}$, Lydia Whitaker $^{\mathrm{a}}$, Asifa Majid $^{\mathrm{c}, \mathrm{d}}$ \\ ${ }^{a}$ University of Essex, Colchester, England, United Kingdom \\ ${ }^{\mathrm{b}}$ Universität Osnabrück, Postfach 44 69, 49069 Osnabrück, Germany \\ ${ }^{\mathrm{c}}$ Max Planck Institute for Psycholinguistics, PO Box 310, 6500 AH, Njimegen, The Netherlands \\ ${ }^{\mathrm{d}}$ Donders Center for Brain, Cognition, and Behaviour, Radboud University, Nijmegen, The Netherlands
}

\section{A R T I C L E I N F O}

\section{Article history:}

Received 5 August 2011

Revised 14 June 2012

Accepted 29 June 2012

Available online 12 August 2012

\section{Keywords:}

Facial expression

Emotion

Child-development

Categories

\begin{abstract}
A B S T R A C T
Three studies investigated developmental changes in facial expression processing, between 3 years-of-age and adulthood. For adults and older children, the addition of sunglasses to upright faces caused an equivalent decrement in performance to face inversion. However, younger children showed better classification of expressions of faces wearing sunglasses than children who saw the same faces un-occluded. When the mouth area was occluded with a mask, children under nine years showed no impairment in expression classification, relative to un-occluded faces. An early selective focus of attention on the eyes may be optimal for socialization, but mediate against accurate expression classification. The data support a model in which a threshold level of attentional control must be reached before children can develop adult-like configural processing skills and be flexible in their use of face- processing strategies.
\end{abstract}

(c) 2012 Elsevier B.V. All rights reserved.

\section{Introduction}

Why do young children have difficulty classifying facial expressions of emotion? If basic expressions of some human emotions (plural) (happiness, anger, fear, surprise, sadness and disgust) are generated by innate biological affect programs (Ekman, Friesen, \& Ellsworth, 1972) what skills need to develop in order to interpret these expressions on other people's faces?

By the age of two children appear to understand emotions as internal feelings, separate from their external causes (Wellman, Harris, Banerjee, \& Sinclair, 1995). By three or four they can match two images of expressions of the same emotion and identify emotions from emotional stories (Widen \& Russell, 2003). Yet they consistently over-extend labels like 'happy' or 'sad' to inappropriate expressions, especially when pairs of facial expressions share the same valence (Russell, 1994; Shelley-Tremblay

\footnotetext{
* Corresponding author. Tel.: +44 01206 873710; fax: +44 01206 873598.

E-mail address: robedd@essex.ac.uk (D. Roberson).
}

\& Mack, 1999). Accurate decoding of facial expressions improves only gradually through childhood and continued refinement of categorization has been observed into adolescence (Camras \& Allison, 1985).

Despite intensive investigation, the cause of these difficulties remains unclear (Tanaka, Kay, Grinnell, Stansfield, \& Szechter, 1998; Wallace, Coleman, \& Bailey, 2008). Children might have some particular difficulty in accessing existing emotion categories from facial expressions, either because their knowledge is less accessible in some response modalities ${ }^{1}$ or because children have less stable, more task-dependent classification criteria than adults. Alternatively, the task may demand a sophisticated level of perceptual processing that they have yet to achieve.

Adults are able to process complex configural aspects of faces when judging identity (Bruce, Doyle, Dench, \& Burton, 1991; Rhodes, Brake, \& Atkinson, 1993; Tanaka \& Farah, 1993), and to take multiple features into account

\footnotetext{
1 When verbal and manual responding has been directly contrasted, children often pointed to a correct choice, but said the incorrect one (Zelazo, Frye \& Rapus, 1996).
} 
when judging expressions (Barrett, Lindquist, \& Gendron, 2007).

Adult face processing strategies are complex and flexible, (Maurer, Le Grand, \& Mondloch, 2002), and impaired by face inversion (McKelvie, 1995; Yin, 1969). Crucially, adults are able to switch strategies between face processing tasks so that they can ignore changes in expression when judging identity, and ignore changes in identity when judging expression. Young children appear to be less flexible, so that while they may be able to process face identity independently from facial expressions, changes in identity interferes with their ability to judge emotion from facial expressions (Krebs et al., 2011). The ability to use different processing strategies for the two tasks may appear rather abruptly, if it depends on the achievement of some attentional threshold (e.g. Schwarzer, 2000).

There is considerable debate as to when adult-like flexibility of face processing emerges (Mondloch, Dobson, Parsons, \& Maurer, 2004; Mondloch, Geldart, Maurer, \& Le Grand, 2003). Although young children show a recognition advantage for individual facial features in the context of a whole face (Tanaka et al., 1998), and have adult-like difficulty in identifying the top half of composite faces when the top and the bottom half are misaligned (de Heering, Houthuys, \& Rossion, 2007; Konar, Bennett, \& Sekuler, 2010), they may continue to rely on external features, such as hair, when identifying familiar faces, (Diamond \& Carey, 1986). Children under 7 show no decrement in face identification for inverted faces and rely strongly on a single attribute of the face for a range of judgments (Schwarzer, 2000; Kestenbaum and Nelson, 1990). Adult levels of facial expression decoding may be achieved relatively late because it requires holistic processing so that, for example, a judgment of the emotion displayed by wide open, staring eyes can be modulated by the shape of the mouth in order to distinguish fear from either pleasant or unpleasant surprise, (Farah, Wilson, Drain, \& Tanaka, 1998).

Problematic for developmental investigations of these issues is the fact that image manipulations that disrupt contextual aspects of identity or expression, all result in further impairments of performance on a task on which young children already make frequent errors. Transformations that disrupt the horizontal 'barcode' sequence of the face such as misalignment of two halves of the face, or part / whole changes, result in disproportionate impairment of adult face processing (Dakin and Watt, 2009), increase task difficulty and distort the expressions that children are being asked to classify. This limits their effectiveness in investigating the causes of children's prolonged difficulties in decoding facial expressions of emotion and increases the difference between adults' and children's performance (see Mondloch et al. (2004), Tanaka et al. (1998) or Valentine (1995) for discussions of these issues).

In the present studies, a more ecologically valid manipulation was used to explore the perceptual processing of facial expressions of emotion. In one manipulation, sunglasses were added to images of facial expressions. This condition should have obliged participants of all ages to shift their attention from the eyes, and instead to attend to the mouth. In the second, the addition of masks obscured the mouth area of the same faces.
If young children's difficulties in expression processing arise because they are unable to process expression as an independent attribute of faces, no systematic reduction in performance should occur. If they arise through selective attention to the eye area in faces (Schwarzer \& Zauner, 2003), and lack the attentional control to attend to more informative features (e.g. the mouth), then the addition of sunglasses should improve expression classification, but the addition of surgical masks should harm it, relative to the full-face condition. If participants are processing configural aspects of the face, similar reductions in accuracy should occur from either the addition of sunglasses or of masks.

Since occlusion of any part of the face reduces the available feature information, as well as disrupting configural processing, in Experiment 1, the effect of sunglasses was compared to inversion in adults, 9-10 year olds and 5-6 year olds.

\section{Experiment 1}

Experiment 1 compared children's and adults' processing of facial expressions of emotion with upright face, inverted faces and faces with sunglasses occluding the eye area. The addition of sunglasses disrupts both configural and featural processing. However, if 5-6 year-olds do not yet use configural processing, then inversion of the fullface should not disrupt their recognition of emotional expressions, but the addition of sunglasses should, because they obscure the preferred feature. Such disruption should be particularly evident for angry faces, because happy faces can still be accurately classified on the basis of the mouth alone. The difference between conditions should be reduced in older children, who should have at least begun to process the faces configurally and should be minimal in adults, for whom equal disruption is predicted.

\subsection{Method}

\subsubsection{Participants}

Twenty 5-6 year-olds ( 8 females, mean age 5 years 7 months) and twenty 9-10 year-olds ( 11 females, mean age 9 years 6 months) recruited with parental consent from local schools in Essex and 20 adult students from the University of Essex (11 females, mean age 21 years) took part in Experiment 1. All had normal or correctedto-normal eyesight.

\subsubsection{Stimuli}

Photographs of a male and a female model posing five expressions of emotion (happiness, surprise, anger, fear and sadness) were taken from the set of images created by Montagne and colleagues and form the basis for "The Emotion Recognition Task' (Montagne, Kessels, De Haan, \& Perrett, 2007). The set are well validated and have been used in a wide range of published neuropsychological and developmental research. All the images were cropped to remove external features and were shown in a front view, resized to $250 \times 250$ pixels. All faces had been adjusted to match in skin tone, overall brightness, and contrast levels. 

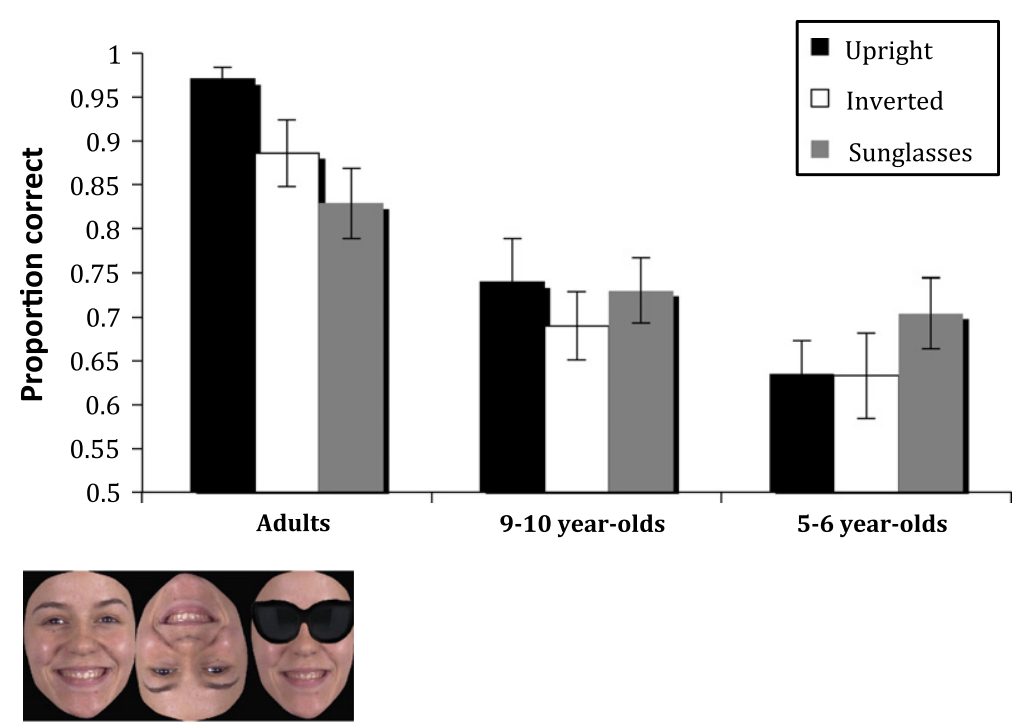

Fig. 1. Proportion of correct classifications of upright or inverted faces with, or without sunglasses, by adults and 5-6-year-olds, with examples of the stimuli (error bars on all figures are standard errors).

Following Widen and Russell (2008), we included only positive examples of 'surprise', an emotion that can be either negatively or positively valence. In a second set of identical images, sunglasses were added (using Adobe Photoshop CS2) covering the eyes in each image.

Each face was shown three times in each of three conditions (full-face upright, full-face inverted, upright face with sunglasses) for a total of 90 trials. The full set of stimuli is shown in Appendix A. The images were presented on a Mac Book Pro laptop computer screen.

\subsubsection{Procedure}

Children were tested in schools, in a quiet corner of the classroom, adults at the University of Essex. All participants completed a short practice session, with feedback, using faces not included in the test phase. In Experiment 1, participants allocated the five $100 \%$ intensity examples of each expression to one of five virtual boxes at the bottom of the computer screen labeled 'happy', 'surprised', 'angry', 'afraid' or 'sad'. Order of stimulus presentation was randomized.

\subsubsection{Results and discussion}

Mean correct classifications for each age group and condition were calculated. A 3 (Age: adult vs. 9-10 vs. 5-6 year-old) $\times 3$ (Condition: upright face vs. inverted face vs. face with sunglasses) mixed design ANOVA, with repeated measures over the second factor revealed a significant effect of age $[F(2,57)=24.95, M S e=.03, p<.05]$, no significant effect of condition $[F(2,114)=1.69, M S e=.03$, $p>.1]$, but a significant interaction $[F(4,114)=2.63$, $M S e=.03, p<.05]$. A Newman-Keuls pairwise comparison of the interaction revealed that for adults, both the addition of sunglasses and the inversion faces significantly impaired classification relative to the upright full-face condition (both $p<.05$ ) but performance in the sunglasses and inversion conditions did not differ. Neither condition impaired classification for 9-10-year-olds, or for 5-6-year- olds compared to the upright full-face condition. Fig. 1 illustrates the interaction.

For adults, the addition of sunglasses disrupted processing at least to the same extent as face inversion. The lack of a significant effect on the performance of 5-6 year-olds cannot be attributed to floor level performance because their classification of expressions shows a slight improvement when the faces had sunglasses, even though there is no effect of face inversion for children at this age. Nine-ten year-olds also show no significant differences between conditions, but an overall performance level that was intermediate between the other two groups.

These results are consistent with the results of investigations of face identity (Diamond \& Carey, 1986) and the resulting hypothesis that children under 12 are not yet competent in configural processing, because even 9-10 year-old children show only a slight trend towards the pattern of adult performance and no significant impairment of classification with inverted faces. An alternative explanation might be that younger children's face processing errors result from a lack of attentional control that would allow them to overcome the strong attraction to the eye area (Haith, Bergman, \& Moore, 1979) in favor of more informative features (e.g. the mouth). This seems unlikely because occluding the eyes with sunglasses does not significantly improve performance even though it removes the preferred focus of attention, so these children may have already learned to attend strategically to the mouth when judging expressions. To investigate children's classification of facial expressions across a broader age range, Experiment 2 used only upright stimuli, either with or without sunglasses in a sorting task.

\section{Experiment 2}

Experiment 2 used a sorting task in which children's classification behavior has been shown to improve 
gradually (Widen \& Russell, 2003, 2008) and that could be completed by preschool children, as well as older children and adults. In the study children and adults were asked to explicitly group faces by expression.

\subsection{Method}

\subsubsection{Participants}

Forty 3-4 year-olds (25 female; mean age 3 years 10 months), forty 5-6 year-olds (19 female; mean age 5 years 8 months), forty 7-8 year-olds (13 female; mean age 7 years 5 months), and forty 9-10 year-olds (18 females; mean age 9 years 7 months) recruited with parental consent from local schools in Essex and 40 adult students from the University of Essex (24 females, mean age $=21.6$ ) participated in Experiment 2 .

\subsubsection{Stimuli}

Each of the ten emotional faces used in Experiment 1 was morphed with an image of the same individual posing a neutral expression to create blends at 3 levels of emotional intensity: $100 \%, 80 \%$, or $60 \%$ of the emotional expression. In this way two sets of fifteen images (one male, one female) were created. An additional set of 10 face images ( 6 female, 4 male), captured from the Internet were used in the practice session in Experiment 2. These images differed in hair color. Five had light colored hair and 5 had dark colored hair. As in Experiment 1, all faces were shown in front view, resized to $250 \times 250$ pixels and adjusted to match in skin tone, brightness, and contrast levels. Four cardboard houses, painted in different colors but otherwise identical, with large 'chimney' slots in the roof into which children could 'post' the laminated images were also used in Experiment 2.

\subsubsection{Procedure}

Participants were tested individually in quiet surroundings. Each child completed two practice sessions before the test phase. The practice sessions involved sorting pictures of people according to the model's hair color (dark or light). The experimenter $(E)$ put two cardboard houses on the table in front of the child. The $E$ pointed to one house and said, "This house is for people with dark hair", and pointed the other house and said, "This house is for people whose hair is not dark." Then the E showed the practice pictures one-by-one to the child and asked, "Which house should this person be in? The house for people with dark hair or the other house for people whose hair isn't dark?"

If the child gave the wrong answer, the E corrected the wrong choice and explained the correct decision referring to hair color. This procedure was repeated for each picture. The second practice session involved selecting the pictures of people with blonde hair. Order of hair color was counterbalanced across children.

The testing phase followed immediately after the practice session. The houses used for the practice phase were replaced with new ones and the experimenter said, "Now we will play a new game. In these houses there are parties for people who are feeling different things. The party in this house is for people who are feeling happy (disgusted, angry, sad, afraid, or surprised)." The experimenter then pointed to the second house and said, "The party in this house is for everyone else who is not happy (...)." All nineteen images were shown one by one to the child in random order, and the children were asked, "Is this person happy (...)? Which house party should he/she be in?" Half the children in each age group saw female faces and the other half saw male faces. The presentation order of the emotions was randomized across children, and every time they moved on to sort different emotion the houses were removed from the table briefly. Adult participants were shown the nineteen faces one by one, and instructed to sort each face into a target expression (e.g. happy) or otherwise. There were no practice trials, and no feedback was given. Half the participants sorted the female set and the other half sorted the male stimuli set. The order of the target emotion was randomized for each participant.

\subsubsection{Results}

For each trial there were three correct inclusions (60\%, $80 \%$ and $100 \%$ intensity of the target emotion) and 12 correct exclusions. The proportion of correct responses (inclusions and exclusions) was recorded for each house party. A five (age: 3-4 years, 5-6 years, 7-8 years, 9-10 years, adult) $\times$ by three (intensity: $60 \%, 80 \%, 100 \%) \times$ two (condition: full face vs. sunglasses) mixed design ANOVA on mean correct responses, with repeated measures over the last two factors, revealed significant effects of Age $[F(4,190)=22.64, M S e=.08, p<.01]$, but not of Condition $[F(1,190)=3.07, M S e=.01, p>.05]$, as well as a significant interaction between Age and Condition $[F(4,190)=5.76$, MSe $=.01, p<.01]$. A Newman-Keuls pairwise comparison of the interaction revealed that for 3-4 year-olds classifications of expressions was significantly better in the sunglasses condition than in the full-face condition $(p<.01)$. For 5-6 year-olds and 7-8 year-olds there was no significant difference between classification in the two conditions for any emotion, but for 9-10 year-olds and adults classification of full faces was significantly better than in the sunglasses condition (see Fig. 2).

To further examine the differential effect of sunglasses on classification of expressions across the five age groups, we compared condition (full face vs. sunglasses) for each Emotional expression (Happy, Surprised, Angry, Sad, Afraid) to establish the effect of sunglasses on each age group's sorting of individual expressions. ${ }^{2}$ Three-four year-olds showed significantly more accurate classification of happy, surprised and sad faces in the sunglasses condition than in the full-face condition (all $p<.05$ ). Five- six year-olds showed significantly better discrimination of happy faces in the full-face than in the sunglasses condition $(p<.05)$. In contrast, 7-8 year-olds showed significantly better discrimination of angry faces in the full-face condition than in the sunglasses condition $(p<.01) ; 9-10$ year-olds showed significantly more accurate classification of both fearful and angry faces in the full-face compared to the sunglasses condition (both $p<.01$ ). Adults showed significantly more

\footnotetext{
${ }^{2}$ We report here only significant differences.
} 


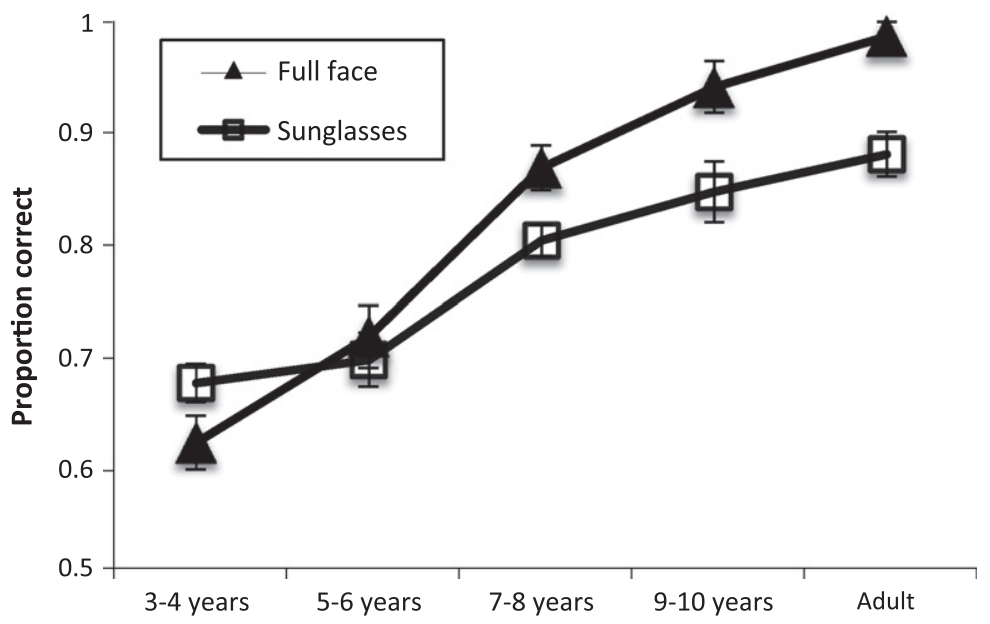

Fig. 2. Proportion of facial expression stimuli with or without sunglasses, sorted correctly by Adults, 5-6-year-olds and 3-4-year-olds.

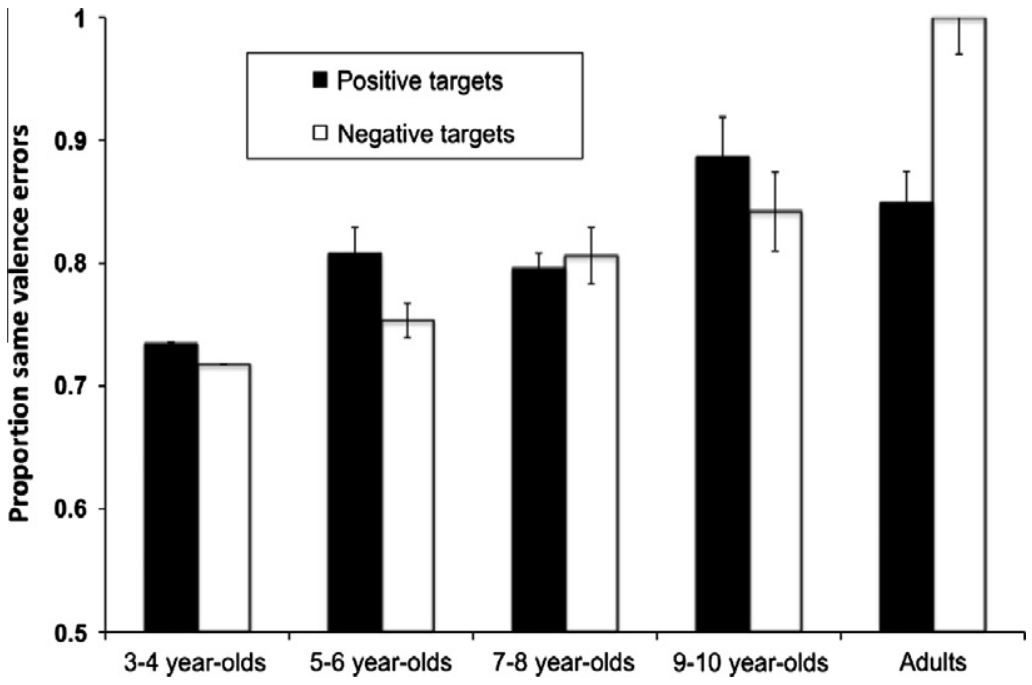

Fig. 3. Breakdown of sorting accuracy data in Fig. 2 by emotion.

accurate classification in the full-face condition than in the sunglasses condition for all emotional expressions $(p<.05)^{3}$.

Within the sorting task, we also investigated participants' sensitivity to different levels of intensity of emotion (Law Smith, Montagne, Perrett, Gill, \& Gallagher, 2010), comparing classification of morphs that contained $60 \%$, $80 \%$ or $100 \%$ of each expression. There was an overall linear improvement in classification with age, such that adults and 9-10-year-olds were equally accurate in sorting all levels of intensity for all the emotions tested. Both 7-8 and 5-6 year-olds showed equal accuracy with $80 \%$ and $100 \%$ levels, but significantly worse performance with $60 \%$ intensity ( $p<.05$ for all expressions). Three-four year-olds showed significantly reduced sensitivity to both

\footnotetext{
3 The breakdown of performance by emotion is reported only for Experiment 2, as there were no significant differences between expressions in the effect of the manipulation in Experiments 1 and 3.
}

$80 \%$ and $60 \%$ morphs, for all faces and especially for angry faces $(p<.01$, all other $p<.05)$.

Finally, we also analyzed the type of errors made by all participants to assess the reported tendency of young children to initially group emotions only by valence as either positive or negative. While the number of errors decreased linearly with age, for all ages, distractors with the same valence as the target expression were mistakenly categorized in the target category significantly more often than distractors with the opposite valence (see Fig. 3).

\subsubsection{Discussion}

Classification of facial expressions of emotion became more acute with development, so that by the age of 9-10 children were as accurate as classifying morphed expressions that contained only $60 \%$ of a target emotion (blended with $40 \%$ of a neutral face) as they were with faces displaying $100 \%$ of the target emotion. Accuracy increased linearly with age and inclusion errors diminished over time in a 
systematic way, so that older children and adults made fewer errors and only confused expressions that had the same valence as the target expression (negative or positive). Alongside these general refinements, there was a systematic change in the effect produced by the addition of sunglasses to the faces. In the youngest age children, sunglasses appeared to facilitate classification of expressions. Between 5 and 8 years of age there was no difference in classification accuracy between full-face stimuli and those wearing sunglasses. For older children and adults, the addition of sunglasses resulted in increased errors. That pattern is consistent with the gradual development of configural processing (e.g. Diamond \& Carey, 1986) in facial expression processing in which 3-year-olds show a pattern of broad classification by valence (positive vs. negative) that is heavily reliant on featural processing, sensitive only to expressions at $100 \%$ intensity and improves when less diagnostic features, which attract preferential attention (e.g. eyes) are hidden from view. This pattern of performance could not result from floor level performance at this age, because 3-4 year-olds who sorted faces with sunglasses made significantly fewer category errors than those who did not.

The pattern of performance observed here is also compatible with a model in which younger children lack attentional control, and are unable to look away from the eye region, which preferentially attracts attention from the earliest age. The improvements seen in their classification of faces with sunglasses could arise because the sunglasses obscure the preferred feature and therefore attention is perforce directed to the mouth region. Experiment 3 sought to distinguish these two potential causes of increased accuracy in the sunglasses condition, by presenting faces in which the mouth region was masked.

\section{Experiment 3}

Masking the mouth region should disrupt configural processing to the same extent as occluding the eyes, so a similar impairment of performance would be predicted from the addition of masks if children's changing performance reflects the development of configural processing. However, if the pattern of performance in Experiment 2 reflects only the development of attentional control that allows children to direct attention away from the eyes, masking the mouth region should impair classification in all age groups, relative to the full-face or the sunglasses condition, but the effect should be least in the youngest children and grow larger as age increases and children are better able to target their attention to key features of an image.

\subsection{Method}

\subsubsection{Participants}

Twenty 3-4 year-olds ( 9 female; mean age 3 years 7 months), twenty 5-6 year-olds (11 female; mean age 5 years 6 months), twenty $7-8$ year-olds ( 11 female; mean age 7 years 8 months), and twenty $9-10$ year-olds ( 8 females; mean age $=9$ years 7 months) recruited with parental consent from local schools in Essex and 20 adult students from the University of Essex (11 females, mean age $=22.4$ ) participated in Experiment 3 .

\subsubsection{Stimuli and procedure}

The stimuli and procedure were identical to the sunglasses condition in Experiment 2, except that, instead of sunglasses, the faces were adapted so that dark masks occluded the mouth area on each face (see Appendix A).

\subsubsection{Results and discussion}

Classifications were scored in the same way as in Experiment 2. The proportion of correct responses (inclusions and exclusions) was recorded for each house party. A five (age: 3-4 years, 5-6 years, 7-8 years, 9-10 years, adult) $\times$ by three (intensity: $60 \%, 80 \%, 100 \%) \times$ two (condition: full- face vs. mask) mixed design ANOVA on mean

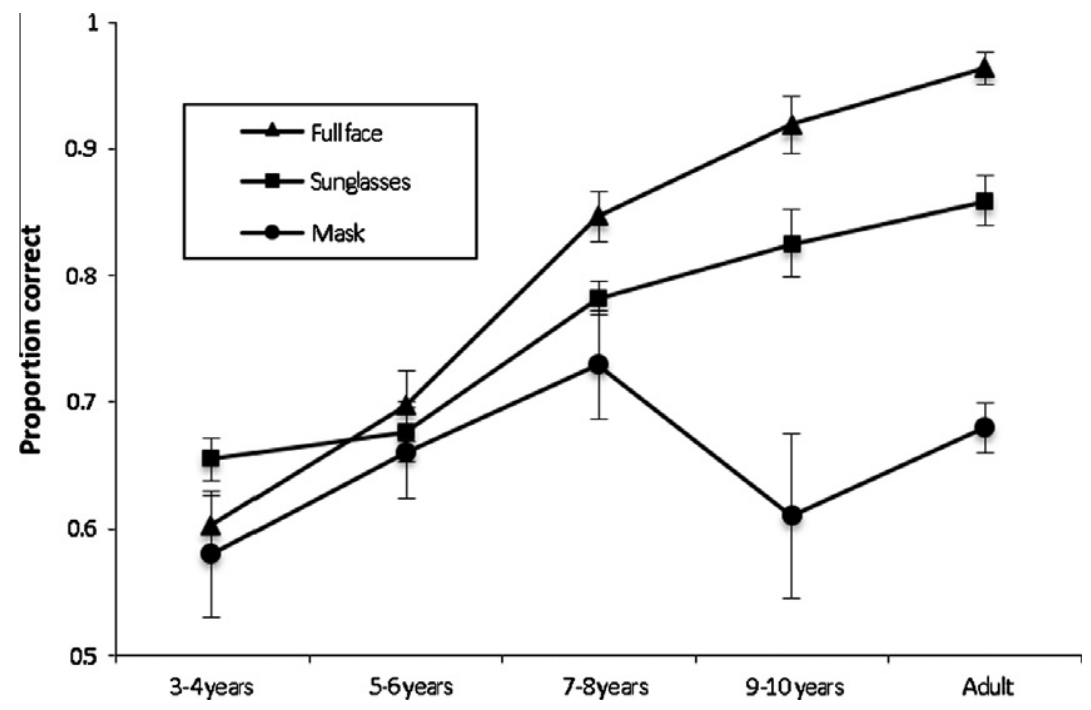

Fig. 4. Proportion of errors to distractors of the same valence as the target (positive/negative) by all participants. 
correct responses, with repeated measures over the last two factors, for the five expressions with masks to the full-face condition of Experiment 2. There were significant effects of Age $[F(4,190)=9.74, \quad M S e=.03, \quad p<.01]$, Condition $[F(1,190)=12.13, M S e=.04, p<.01]$, as well as a significant interaction between Age and Condition $[F(4,190)=15.06$, MSe $=.04, p<.01]$. A Newman-Keuls pairwise comparison of the interaction revealed that for 3-4 year-olds, 5-6 year-olds and 7-8 year-olds there was no significant difference between classification in the two conditions, but classification of full faces by $9-10$ year-olds and adults was significantly better than in the masked condition (see Fig. 4).

When the mouth region of the face was obscured, classifications by the youngest three age groups were as good as by those children in the full-face condition of Experiment 2. This suggests that children under nine preferentially attend to the eye area, even when the full face is visible. If facial expression decoding relies on the eyes alone until at least the age of 7 (Schwarzer, 2000) then occluding another part of the face will have little effect on performance. However, for older children and adults occluding the mouth significantly impairs performance. This is consistent with an emergent ability to change strategies when processing faces for emotion or identity, taking account of different contextual factors for each type of judgment (Barrett, Mesquita, \& Gendron, 2011).

\section{General discussion}

In Experiment 1 neither inversion nor the addition of sunglasses affected the classification of 5-6 year olds, but both manipulations appeared to be equally successful in disrupting the accurate decoding of facial expressions by 9-10 year olds and adults. The effect of both manipulations on older children and adults is consistent with the hypothesis that configural processing is both slow to develop (e.g. Diamond \& Carey, 1986) and disrupted by both processes. This need not imply that 7-year-olds are incapable of processing any configural information about faces. Rather, children under 8 years old may strategically focus on a single feature - the eyes - to process both face identity and facial expressions. Kestenbaum and Nelson (1992) found neurophysiological differences between 7 year-olds and adults in face processing tasks, and Meaux, Gillet, Bonnet-Brilhault, Barthélémy, and Batty (2011), found that normally developing 8-year-olds had difficulty discriminating emotions from isolated features (eyes or mouth) compared to when the whole face was visible, but autistic individuals, whose expression processing was less advanced, did not.

The predisposition to attend to the eye region, that appears to be present within a few hours of birth, (BaronCohen, Wheelwright, \& Jolliffe, 1997; Haith et al., 1979) and engages emotional arousal systems associated with the 'social' brain (Senju \& Johnson, 2009) may impair children's capacity to identify facial expressions because, out of context, the eyes give ambiguous information about emotional expressions (Jack, Blais, Scheepers, Schyns, \& Caldara, 2009). This bias may result in the slow development of configural face processing and the prolonged error- prone classification of facial expressions. The addition of sunglasses to images of facial expressions allows observers to overcome this early bias to attend preferentially to the eyes, leading to better performance by the youngest children. Consistent with this, the addition of masks covering the mouth area of the same stimuli did not impair performance for children aged between 3 and 8 years of age. If children at that age are attending only to the eye region, then occluding another area of the face should not impair performance. Increasing flexibility of face processing strategies allows older children to attend to the mouth region to decode facial expressions, resulting in increased accuracy when the full face is visible.

However, increased attentional control alone cannot account for the pattern of performance in Experiment 3. When faces were masked classification improved between 3-4 and 7-8 years, but dropped abruptly between the ages of 7-8 and 9-10. The abrupt change in the effect of occlusion is consistent with a change from a feature-based to a configural processing strategy at around 9 years of age for facial expressions as well as for face identity. Adults, who routinely process configural aspects of facial expressions as well as face identity, show similar effects of the manipulation to the older children. There may be a threshold of attentional control that must be attained in order for children to achieve flexible face processing strategies, which in turn may facilitate the adoption of configural processing.

Together these experiments demonstrate, for the first time, that the addition of sunglasses leads to improved performance in very young children. The paradigm has the advantage of avoiding floor effects, which may have led to conflicting results in developmental studies of face processing that used inversion or miss-alignment for investigating face processing. It should be particularly useful in studies of emotion processing in specialist populations (such as individuals with Autistic Syndrome Disorder; Pollak, Messner, Kistler, \& Cohn, 2008), for whom misalignment or inversion may result in failure to process the stimulus as a face. The use of sunglasses or masks on faces offers a new, effective and ecologically valid means of investigating the time-course of changes in face processing strategies. Future research may establish whether it is equally effective in studies of face identity processing.

\section{Appendix A}

The full set of faces with, and without sunglasses used in Experiment 2. Half the participants at each age sorted the 15 male faces and half sorted the female faces.

Experiment 1 used only the 100\% emotional faces displayed in the left column for each of the two models: 10 full-face upright, 10 full-face inverted and 10 upright with sunglasses. 
Without sunglasses:

Male

$100 \%$
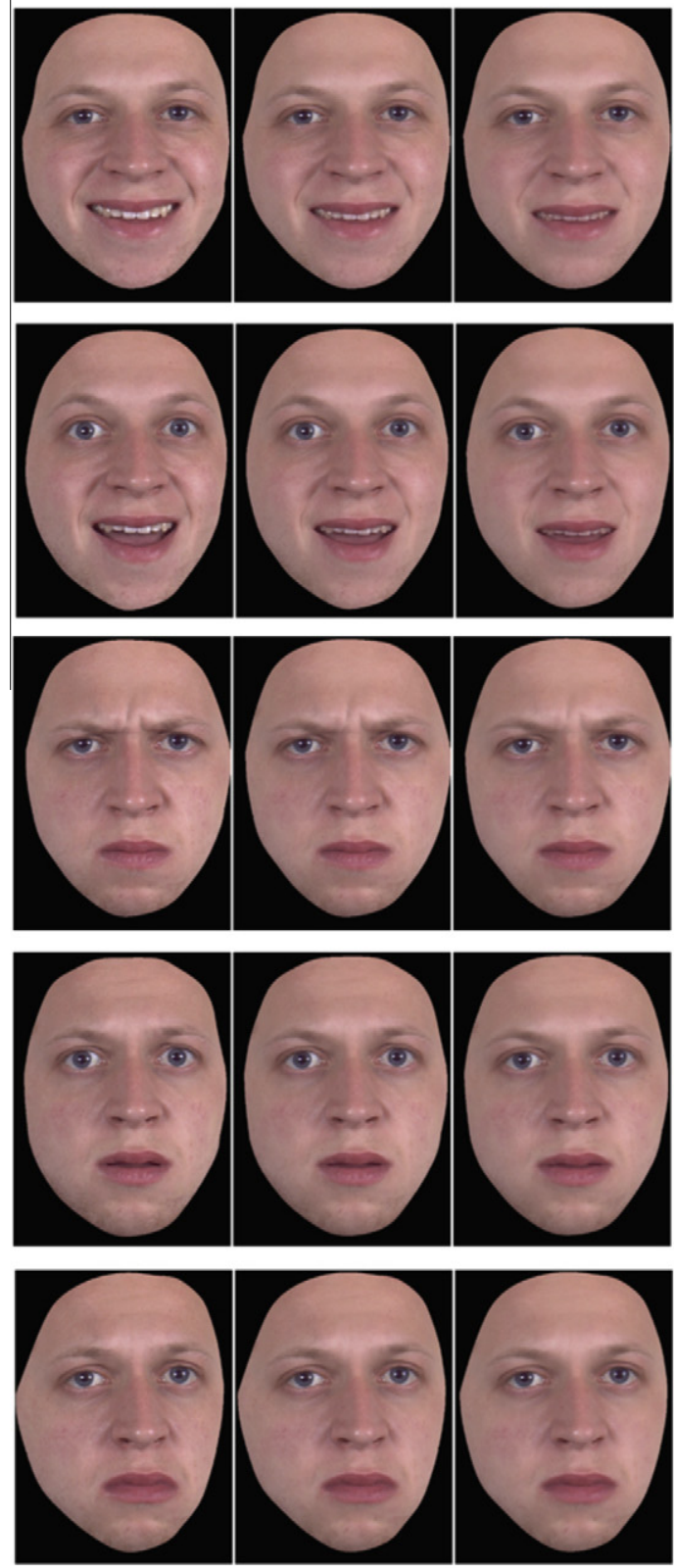

Female

$100 \%$

$80 \%$

$60 \%$
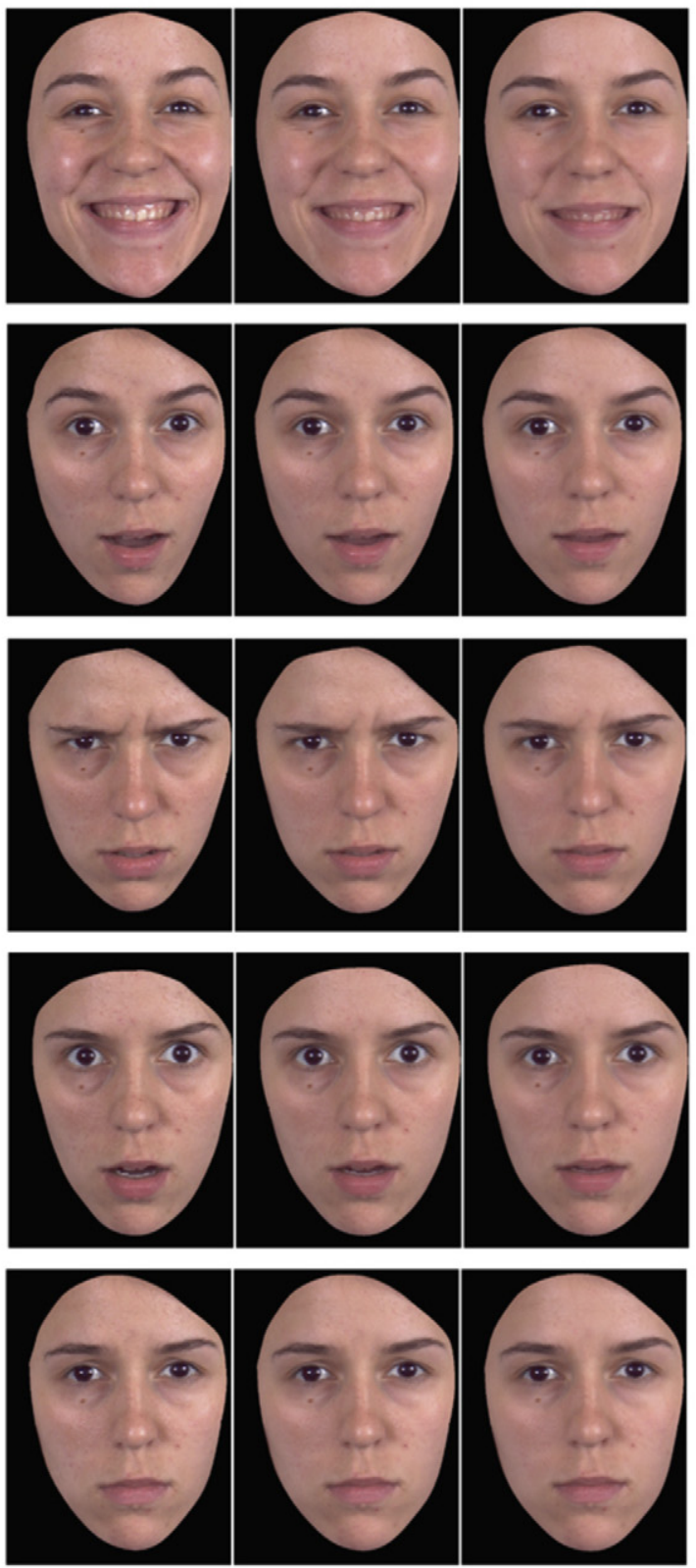

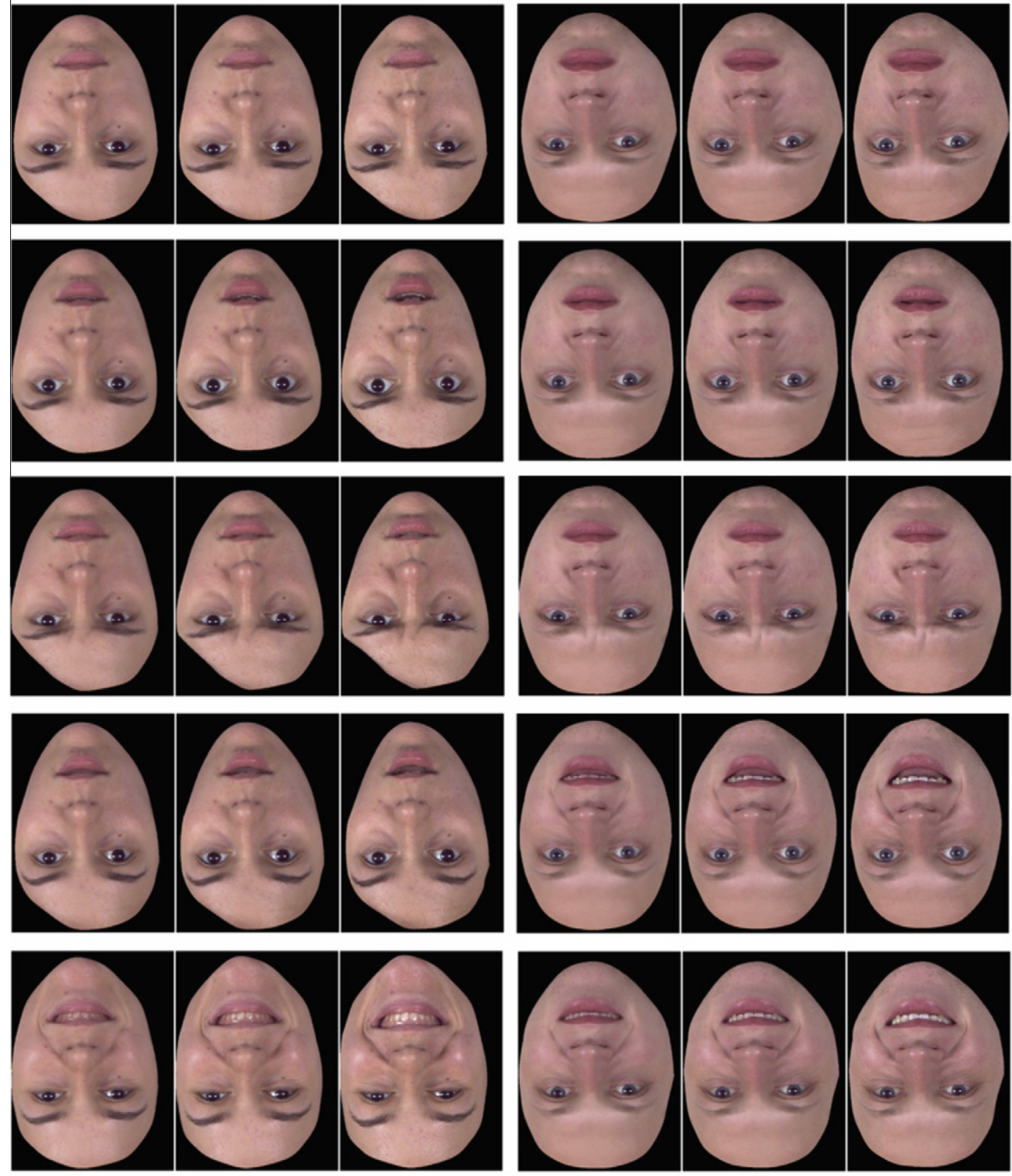
With sunglasses:

Male

$100 \%$
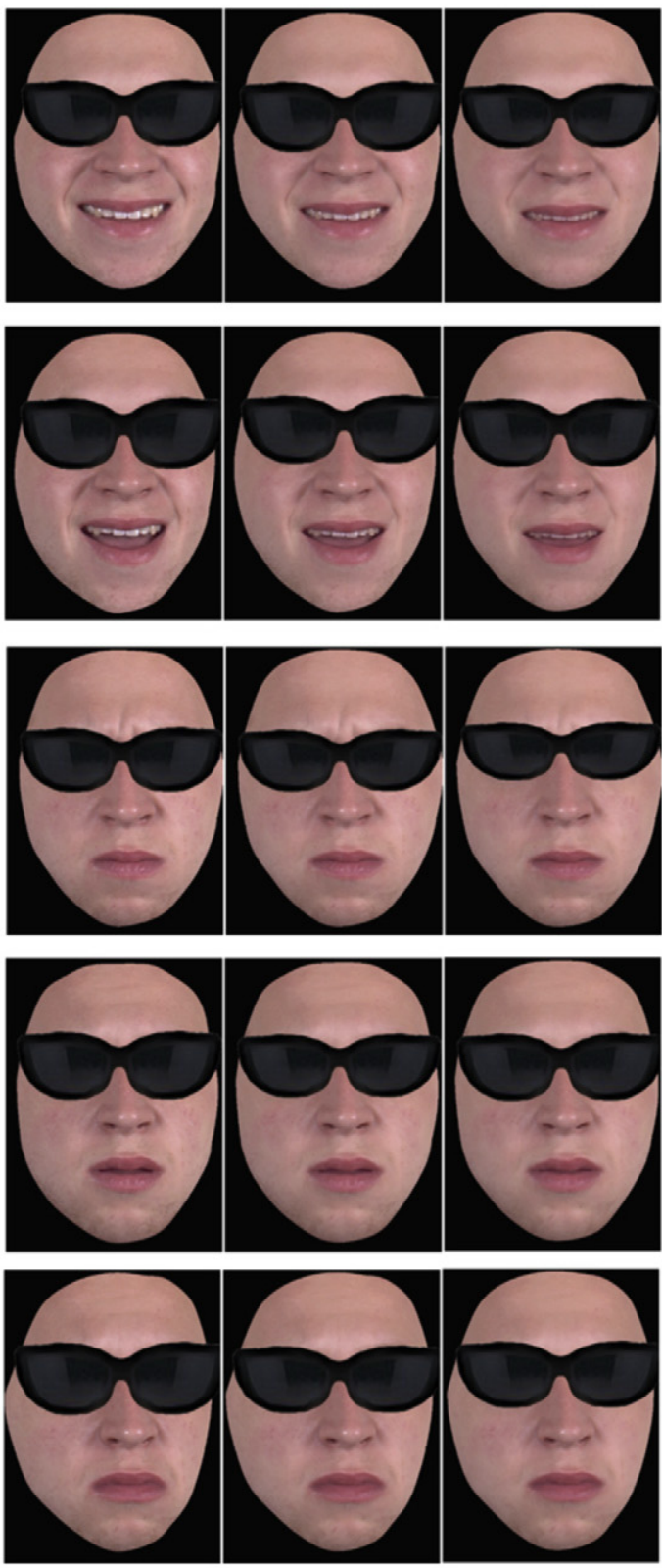

Female

$100 \%$

$80 \%$

$60 \%$
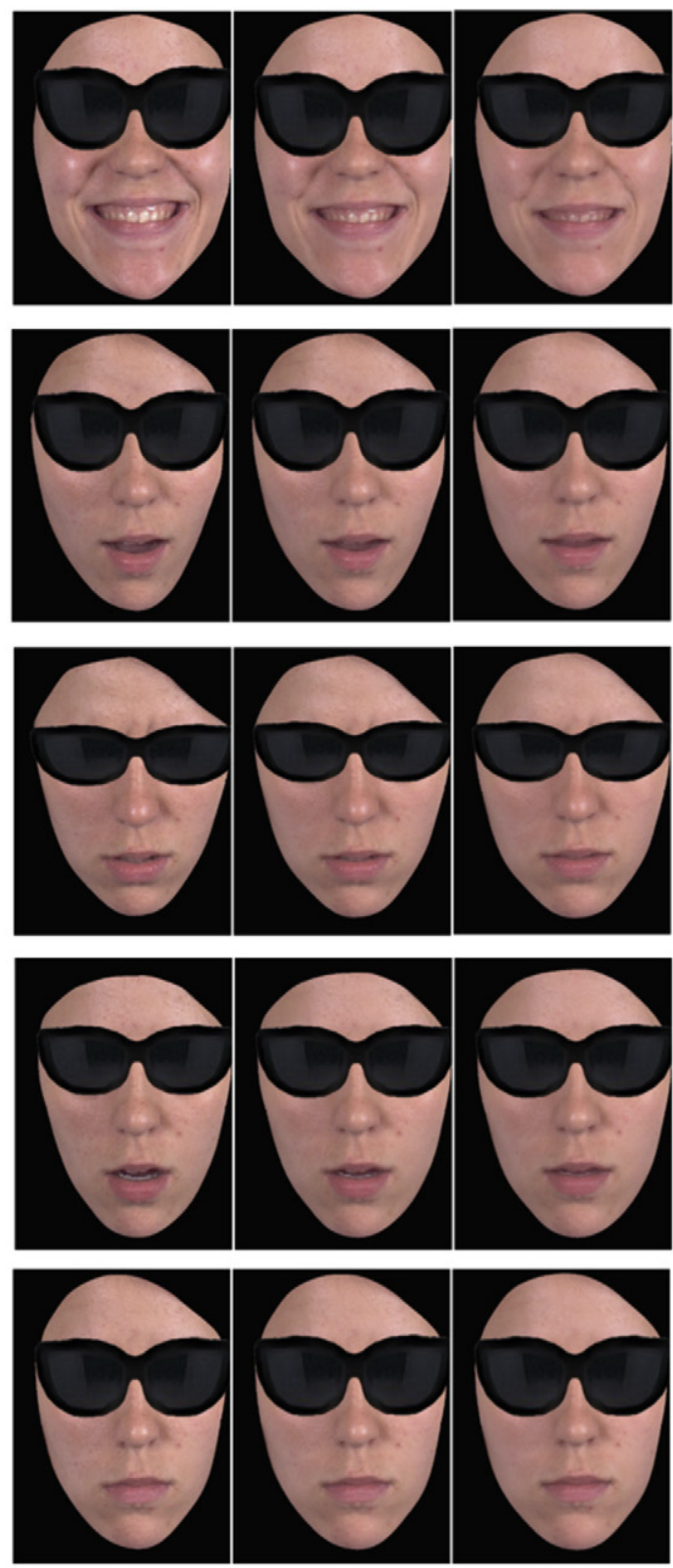

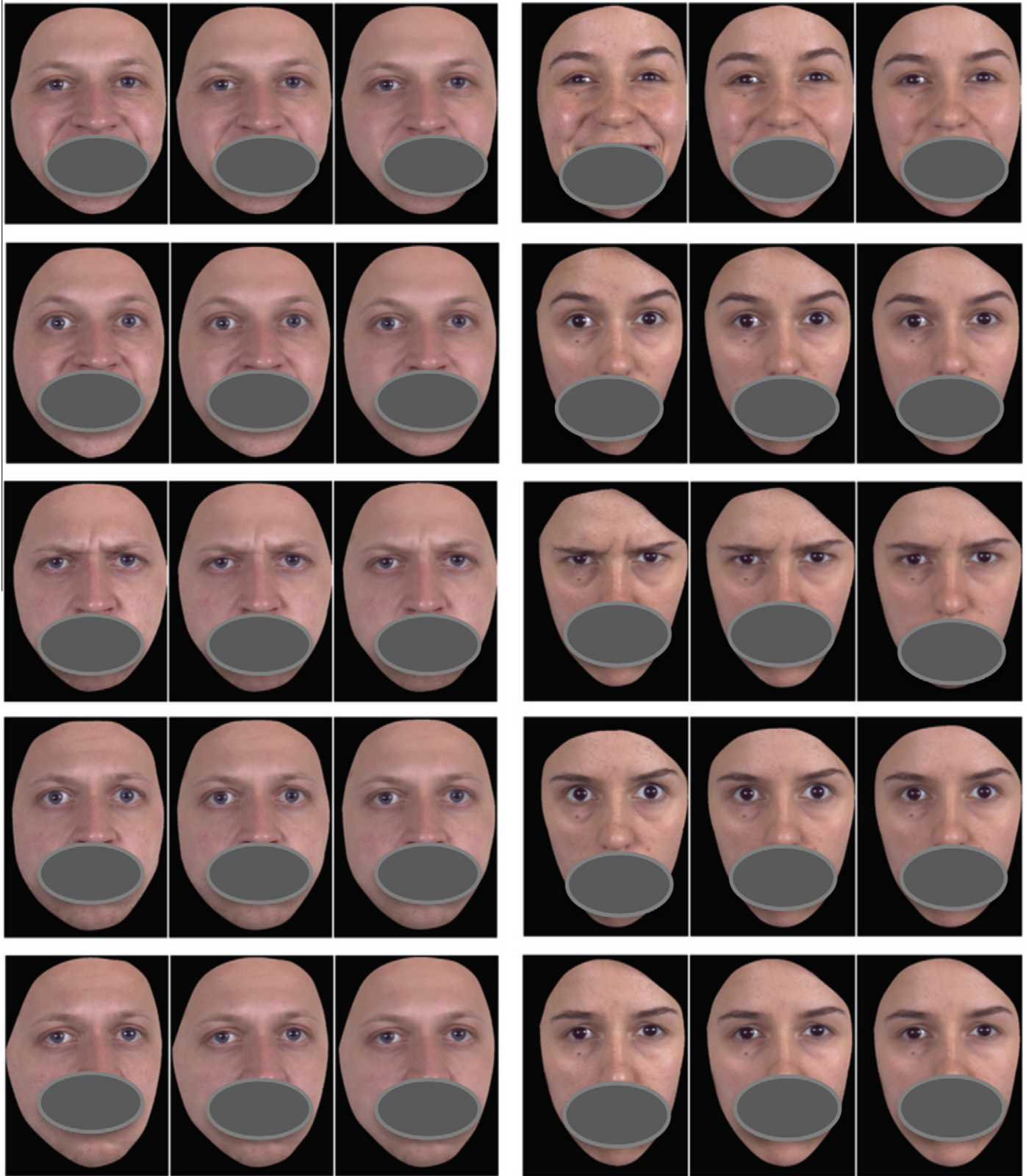

\section{References}

Baron-Cohen, S., Wheelwright, S., \& Jolliffe, T. (1997). Is there a "language of the eyes"? Evidence from normal adults, and adults with autism or Asperger syndrome. Visual Cognition, 4, 311-331.

Barrett, L. F., Lindquist, K. A., \& Gendron, M. (2007). Language as context for the perception of emotion. Trends in Cognitive Science, 11, $327-332$.

Barrett, L. F., Mesquita, B., \& Gendron, M. (2011). Context in emotion perception. Current Directions in Psychological Science, 20, 286-290.

Bruce, V., Doyle, T., Dench, N., \& Burton, M. (1991). Remembering facial configurations. Cognition, 38, 109-144.

Camras, L. A., \& Allison, K. (1985). Children's understanding of emotional facial expressions and verbal labels. Journal of Nonverbal Behavior, 9, 84-94.

Dakin, S. C., \& Watt, R. J. (2009). Biological "bar codes" in human faces. Journal of Vision, 9(4:2), 1-10.

de Heering, A., Houthuys, S., \& Rossion, B. (2007). Holistic face processing is mature at 4 years of age: evidence from the composite face effect. Journal of Experimental Child Psychology, 96, 57-70.

Diamond, R., \& Carey, S. (1986). Why faces are and are not special: An effect of expertise. Journal of Experimental Psychology: General, 115, 107-117.

Ekman, P., Friesen, W. V., \& Ellsworth, P. C. (1972). Emotion in the human face. Guidelines for research and an integration of findings. New York: Pergamon Press.

Farah, M. J., Wilson, K. D., Drain, M., \& Tanaka, J. N. (1998). What is "special" about face perception? Psychological Review, 105, 482-498.

Haith, M. M., Bergman, T., \& Moore, M. J. (1979). Eye contact and face scanning in early infancy. Science, 198, 853-855. 
Jack, R. E., Blais, C., Scheepers, C., Schyns, P. G., \& Caldara, R. (2009) Cultural confusions show that facial expressions are not universal. Current Biology: CB, 19, 1543-1548.

Kestenbaum, R., \& Nelson, C. A. (1990). The recognition and categorization of upright and inverted emotional expressions by 7-month-old infants. Infant Behavior and Development, 13, 497-511.

Kestenbaum, R., \& Nelson, C. A. (1992). Neural and behavioral correlates of emotion recognition in children and adults. Journal of Experimental Child Psychology, 54, 1-18.

Konar, Y., Bennett, P. J., \& Sekuler, A. B. (2010). Holistic processing is not correlated with face-identification accuracy. Psychological Science, 21, 48-53.

Krebs, J., Biswas, A., Pascalis, O., Kamp-Becker, I., Remschmidt, H. H., \& Schwarzer, G. (2011). Face processing in children with autism spectrum disorder: Independent or interactive processing of facial identity and facial expression? Journal of Autism $\mathcal{E}$ Developmental Disorders, 41, 796-804.

Law Smith, M. J., Montagne, B., Perrett, D. I., Gill, M., \& Gallagher, L. (2010) Detecting subtle facial emotion recognition deficits in highfunctioning Autism using dynamic stimuli of varying intensities. Neuropsychologia, 48, 2777-2781.

Maurer, D., Le Grand, R., \& Mondloch, C. J. (2002). The many faces of configural processing. Trends in Cognitive Sciences, 6, 255-260.

McKelvie, S. J. (1995). Emotional expression in upside-down faces: Evidence for configurational and componential processing. The British Journal of Social Psychology, 34, 325-334.

Meaux, E., Gillet, P., Bonnet-Brilhault, F., Barthélémy, C., \& Batty, M (2011). Atypical perception processing and facial emotion disorder in autism. Encephale, 37, 371-378.

Mondloch, C. J., Dobson, K. S., Parsons, J., \& Maurer, D. (2004). Why 8-yearolds cannot tell the difference between Steve Martin and Paul Newman: Factors contributing to the slow development of sensitivity to the spacing of facial features. Journal of Experimental Child Psychology, 89, 159-181.

Mondloch, C. J., Geldart, S., Maurer, D., \& Le Grand, R. (2003). Developmental changes in face processing skills. Journal of Experimental Child Psychology, 86, 67-84.

Montagne, B., Kessels, R. P. C., De Haan, E. H. F., \& Perrett, D. I. (2007). The Emotion Recognition Task: A paradigm to measure the perception of facial emotional expressions at different intensities. Perceptual and Motor Skills, 104, 589-598.

Pollak, S., Messner, M., Kistler, D. J., \& Cohn, J. F. (2008). Development of perceptual expertise in emotion recognition. Cognition, 110, 242-247.

Rhodes, G., Brake, S., \& Atkinson, A. P. (1993). What's lost in inverted faces? Cognition, 47, 25-57.

Russell, J. A. (1994). Is there universal recognition of emotion from facial expression - A review of the cross-cultural studies. Psychological Bulletin, 115, 102-141.

Senju, A., \& Johnson, M. H. (2009). The eye contact effect: Mechanisms and development. Trends in Cognitive Science, 13, 127-134.

Schwarzer, G. (2000). Development of face processing: The effect of face inversion. Child Development, 71, 391-401.

Schwarzer, G., \& Zauner, N. (2003). Face processing in 8-month-old infants: Evidence for configurai and analytical processing. Vision Research, 43, 2783-2793.

Shelley-Tremblay, J. F., \& Mack, A. (1999). Attention modulates metacontrast masking. Psychological Science, 10, 508-515.

Tanaka, J. W., \& Farah, M. J. (1993). Parts and wholes in face. Quarterly Journal of Experimental Psychology, 46, 969-993.

Tanaka, J. W., Kay, J. B., Grinnell, E., Stansfield, B., \& Szechter, L. (1998) Face recognition in young children: When the whole is greater than the sum of its parts. Visual Cognition, 5, 479-496.

Valentine, T. (1995). Cognitive and computational aspects of face recognition: Explorations in face space. London: Routledge.

Wallace, S., Coleman, M., \& Bailey, A. (2008). Face and object processing in autism spectrum disorders. Autism Research, 1, 43-51.

Wellman, H. M., Harris, P. L., Banerjee, M., \& Sinclair, A. (1995). Early understanding of emotion: Evidence from natural language. Cognition E' Emotion, 9, 117-149.

Widen, S. C., \& Russell, J. A. (2003). A closer look at preschoolers' freely produced labels for facial expressions. Developmental Psychology, 39, $114-128$

Widen, S. C., \& Russell, J. A. (2008). Children acquire emotion categories gradually. Cognitive Development, 23, 291-312.

Yin, R. K. (1969). Looking at upside-down faces. Journal of Experimental Psychology, 81, 141-145.

Zelazo, P. D., Frye, D., \& Rapus, T. (1996). An age-related dissociation between knowing rules and using them. Cognitive Development, 11, 37-63. 